\title{
A PROOF OF $K$-THEORETIC LITTLEWOOD-RICHARDSON RULES BY BENDER-KNUTH-TYPE INVOLUTIONS
}

\author{
Takeshi Ikeda and Tatsushi Shimazaki
}

\begin{abstract}
The $K$-theoretic Littlewood-Richardson rule due to A. Buch describes the product structure constants for the Grothendieck polynomials of Grassmannian type. We present a simple self-contained proof of the rule by generalizing Stembridge's cancelation argument which was applied for the classical Littlewood-Richardson rule.
\end{abstract}

\section{Introduction}

The Littlewood-Richardson rule (LR-rule) [6] is a combinatorial procedure for computing the structure constants for the multiplication of Schur functions. The same rule also gives the structure constants with respect to the Schubert basis in the cohomology ring of the Grassmann variety. Buch [2] proved an analogous formula in the Grothendieck ring ( $K$-theory) of the Grassmannian. This ring has a distinguished $\mathbb{Z}$-basis formed by the classes of the structure sheaves of Schubert varieties. Buch's rule gives an explicit description for the structure constants of this basis in terms of combinatorial objects called set-valued semistandard tableaux. The purpose of this article is to present a simple self-contained proof of Buch's rule.

Let $n$ be a positive integer. Let $\mathcal{P}_{n}$ denote the set of partitions with at most $n$ non-zero parts, i.e., the set of integer sequences of the form $\lambda=\left(\lambda_{1} \geq \cdots \geq \lambda_{n} \geq 0\right)$. The set $\left\{(i, j) \in \mathbb{N}^{2} \mid 1 \leq i \leq n, 1 \leq j \leq \lambda_{i}\right\}$ is called the Young diagram of $\lambda$. In drawing such diagram, we adopt the convention, as with matrices, that the first coordinate $i$ increase as one goes downwards, and second coordinates $j$ increases as one goes from left to right. We often identify $\lambda \in \mathcal{P}_{n}$ with its Young diagram. The set-valued tableaux generalizes ordinary Young tableaux in that the boxes are labeled with non-empty subsets of $\{1, \ldots, n\}$, but otherwise satisfy the usual requirements that the entries weakly increase within rows and strictly increase within columns (see Section 2.1 for more precise definition). An example of such tableau is given by the following:

$\begin{array}{cccccc}1 & 1 & 1 & 1 & 1 & 1 \\ 2 & 2 & 3 & 3 & 34 & 4 \\ 3 & 4 & 45 & 5 & 5 & \\ 45 & & & & & \end{array}$.

Buch's rule reads exactly the same as does a version of the classical LR-rule expressed in terms of reverse lattice words (see [3], for example).

The Grothendieck polynomials were introduced by Lascoux and Schützenberger as representatives for the structure sheaves of the Schubert varieties in a flag variety

Received by the editors November 1, 2013.

2000 Mathematics Subject Classification. 05E05, 14M15, $19 \mathrm{E} 08$. 
$[4,5]$. Let $S_{\infty}=\bigcup_{m \geq 1} S_{m}$, where $S_{m}$ denotes the symmetric group of degree $m$. For any permutation $w \in S_{\infty}$, the corresponding Grothendieck polynomial $\mathfrak{G}_{w}=$ $\mathfrak{G}_{w}\left(x_{1}, x_{2}, \ldots\right)$ is a polynomial in the ring of polynomials with integer coefficients in the variables $x_{1}, x_{2}, \ldots$ It can be defined by using the so-called isobaric divided difference operators.

In this paper, we consider the Grothendieck polynomials indexed by the Grassmannian permutations. For $\lambda \in \mathcal{P}_{n}$, let $w_{\lambda} \in S_{\infty}$ be a permutation uniquely determined by the condition that $w_{\lambda}(i)=i+\lambda_{n+1-i}$ for $1 \leq i \leq n$ and $w_{\lambda}(i)<w_{\lambda}(i+1)$ for $i \neq n$. The permutation $w_{\lambda}$ is called the Grassmannian permutation for $\lambda$ with descent in position $n$. We denote the corresponding Grothendieck polynomial $\mathfrak{G}_{w_{\lambda}}$ by $G_{\lambda}$, which is a polynomial in $\mathbb{Z}\left[x_{1}, \ldots, x_{n}\right]$. By a result of Buch [2], the polynomial $G_{\lambda}$ is equal to the generating function of the set $\mathcal{T}(\lambda)$ of all set-valued tableaux of shape $\lambda$. We define the weight of $T$ to be $\omega(T)=\left(\omega_{1}(T), \ldots, \omega_{n}(T)\right) \in \mathbb{N}^{n}$, where $\omega_{i}(T)$ denotes the number of $i$ 's in $T$. We let $|T|$ denote the sum of the cardinalities of the sets in the boxes of $T$. Then we have

$$
G_{\lambda}=\sum_{T \in \mathcal{T}(\lambda)} \beta^{|T|-|\lambda|} x^{\omega(T)}, \quad x^{\omega(T)}=x_{1}^{\omega_{1}(T)} \cdots x_{n}^{\omega_{n}(T)},
$$

where $\beta$ is a formal parameter. In the original definition of the Grothendieck polynomials, $\beta$ is set to -1 , which is relevant when we apply the polynomial to the $K$-theory. Note that if we set $\beta=0$, then (1.1) is the well-known tableaux formula for the Schur function $s_{\lambda}$. The polynomial $G_{\lambda}$ represents the structure sheaf of the corresponding Schubert variety in the $K$-theory of the Grassmannian $\operatorname{Gr}\left(n, \mathbb{C}^{n+m}\right)$ for all $m \geq \lambda_{1}$.

Thus the problem is translated into a purely combinatorial one to describe the product $G_{\lambda} G_{\mu}$ as a $\mathbb{Z}$-linear combination of $G_{\nu}$ 's. Buch's proof of the LR-rule uses an insertion algorithm for set-valued tableaux that only works for inserting a single column into a tableau. He also uses non-commutative version of the Grothendieck polynomials in the so-called local plactic algebra.

We follow the same line of Stembridge's proof [8] of the classical LR-rule, where he used the bi-alternant formula along with the semistandard tableaux model for Schur functions and the so-called the Bender-Knuth involutions ([1]) on the set of semistandard tableaux. The involutions were used to make a pair of tableaux corresponding to "unwanted terms" in the final formula. Such terms pairwise cancel by virtue of the sign-reversing property of the involution.

The first ingredient of our argument is a set-valued version the Bender-Knuth involutions. As an application of this construction, we can prove that $G_{\lambda}$ is a symmetric function in $x_{1}, \ldots, x_{n}$. In order to prove the main theorem, we introduce another involution on the set of unwanted tableaux. This second type of involution has no counterpart in non-set-valued case. Combining the two involutions, we can form pairs or 'quadruples' of unwanted tableaux to prove the result. As in the case of Stembridge's proof for Schur functions, our argument proves the following closed expression for $G_{\lambda}$ :

$$
G_{\lambda}=\frac{\operatorname{det}\left(x_{i}^{\lambda_{j}+n-j}\left(1+\beta x_{i}\right)^{j-1}\right)_{n \times n}}{\prod_{1 \leq i<j \leq n}\left(x_{i}-x_{j}\right)} .
$$

Another proof of Buch's LR-rule has been obtained by McNamara [7] by using a deformed version of $G_{\lambda}$ called the factorial Grothendieck polynomials. In [7] a recurrence relation for deformed structure constant was used. 


\section{Results}

2.1. Set-valued semistandard tableaux. Let $\lambda, \mu \in \mathcal{P}_{n}$ be such that $\lambda_{i} \leq \mu_{i}(1 \leq$ $i \leq n)$. Then $\lambda$, considered to be a Young diagram, is a subset of $\mu$. The set-theoretical difference $\mu-\lambda$ (often denoted by $\mu / \lambda$ ) is called a skew Young diagram. Let $\theta$ be a skew Young diagram. A labeling of $\theta$ is a map from the set of all boxes in the skew Young diagram $\theta$ to the set of non-empty subsets of $\{1, \ldots, n\}$. If $A$ and $B$ are two non-empty subsets of $\{1, \ldots, n\}$, we will write $A<B$ if $\max (A)<\min (B)$, and $A \leq B$ if $\max (A) \leq \min (B)$. We define a set-valued semistandard tableau to be a labeling of $\theta$, such that the rows are weakly increasing from left to right and the columns strictly increasing from top to bottom. This condition on a labeling will be referred to as semistandardness. The shape of a set-valued tableau $T$ is the (skew) Young diagram it is labeling of. Let $\mathcal{T}(\theta)$ denote the set of all set-valued semistandard tableaux of shape $\theta$. We simply write a tableau for a set-valued semistandard tableau.

It is natural to extend the definition of Grothendieck polynomial for skew partition $\theta$. Let $G_{\theta}$ denote by the generating function of $\mathcal{T}(\theta)$ defined by $(1.1)$ with $\mathcal{T}(\theta)$ instead of $\mathcal{T}(\lambda)$.

2.2. Bender and Knuth-type involutions. We introduce a set of involutions $\sigma_{1}, \ldots, \sigma_{n-1}$ on $\mathcal{T}(\theta)$, with the property that $\sigma_{i}$ acts by changing certain entries of $T \in \mathcal{T}(\theta)$ from $i$ to $i+1$ and vice versa in such a way that $\omega\left(\sigma_{i}(T)\right)=s_{i} \omega(T)$, where $s_{i}$ exchanges the $i$ th and the $(i+1)$-components of $\omega(T)$. The existence of these involutions proves that $G_{\theta}$ is a symmetric function of $x_{1}, \ldots, x_{n}$.

Let $T$ be a tableau in $\mathcal{T}(\theta)$. An entry $i$ in a box is free if there is no $i+1$ in the box below. An entry $i+1$ in a box is free if there is no $i$ in the box above. Note that if a box contain both $i$ and $i+1$, then the both entries are free. It means that we can define a box to be free if it contains a free entry. For any given row, the free boxes occur in a consecutive columns. Let $B_{1}, \ldots, B_{m}$ be such boxes. Here by consecutive we mean that $B_{i}$ is to the left of $B_{i+1}$ for $1 \leq i \leq m-1$. For $j$ from 1 to $m$, set $A_{j}=T\left(B_{j}\right) \cap\{i, i+1\}$, where $T\left(B_{j}\right)$ denote the set of letters assigned to the box $B_{i}$ by $T$. Note that $A_{j}$ is one of $\{i\},\{i+1\},\{i, i+1\}$. We set

$$
s_{i}(\{i\})=\{i+1\}, \quad s_{i}(\{i+1\})=\{i\}, \quad s_{i}(\{i, i+1\})=\{i, i+1\} .
$$

For $1 \leq j \leq m$, we change the entry of the box $B_{j}$ by replacing the subset $A_{j}$ of $T\left(B_{j}\right)$ with $s_{i}\left(A_{m-j+1}\right)$, letting the entries other than $i, i+1$ unchanged. Let $\sigma_{i}(T)$ denote the obtained labeling of $\theta$ by performing these changes in all rows of $T$. It turns out that $\sigma_{i}(T)$ is semistandard. In fact, $s_{i}\left(A_{m}\right), \ldots, s_{i}\left(A_{1}\right)$ satisfies the semistandardness. The conditions of semistandardness about vertical positions are also satisfied. In fact, one sees that there is at most one $i$-free box for each column of $T$, so there are no problems for such columns. Also it is not difficult to see that the change does not destroys the semistandardness at the $i$-fixed columns.

For example, if $i=4$ the left tableau in the following is changed into the right one and vise-versa:

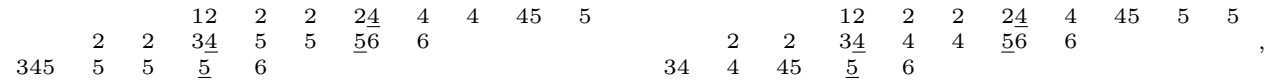

where we underlined the entries which are not free. 
2.3. Column words and $\lambda$-good tableaux. We define the column word of $T \in$ $\mathcal{T}(\theta)$ to be the sequence $c w(T)$ of the integers contained in its boxes when these are read from top to bottom in each column, starting from the rightmost column and moving to left. The integers within a single box are arranged in the decreasing order. Let $c w(T)_{j}$ denote the subword of $c w(T)$ formed by the first $j$ letters in $c w(T)$. For the tableau $T$ in the Introduction, we have $c w(T)_{8}=14143513$. For a word $c w(T)_{j}$ its weight $\omega\left(c w(T)_{j}\right) \in \mathbb{N}^{n}$ is defined naturally by counting the numbers of entries. For example the weight of the above word $c w(T)_{8}$ is $(3,0,2,2,1)$.

Definition 1. Let $\lambda$ be a partition and $\theta$ be a skew diagram. A tableau $T \in \mathcal{T}(\theta)$ is $\lambda$-good if $\lambda+\omega\left(c w(T)_{j}\right)$ is a partition for all $j \geq 1$. Let $\mathcal{T}(\theta)_{\kappa}^{\lambda \text {-good }}$ denote the set of all $\lambda$-good tableaux of shape $\theta$ with weight $\kappa \in \mathbb{N}^{n}$.

Theorem 1. For all $\lambda \in \mathcal{P}_{n}$ and skew diagram $\theta$, we have

$$
G_{\lambda} \cdot G_{\theta}=\sum_{\nu \in \mathcal{P}_{n}} \beta^{|\nu|-|\lambda|-|\theta|} c_{\lambda \theta}^{\nu} G_{\nu},
$$

where $c_{\lambda \theta}^{\nu}$ is equal to the cardinality of the set $\mathcal{T}(\theta)_{\nu-\lambda}^{\lambda \text {-good }}$.

Remark. The description for $c_{\lambda \theta}^{\nu}$ of the above theorem is seemingly different from Buch's rule, while it is quite similar to the description by McNamara [7]. See [7] for the equivalence of those two formulations.

Example. Let $\lambda=(2,1,0), \mu=(2,2,0)$. Then $G_{\lambda} \cdot G_{\mu}$ is expanded as follows: $G_{(4,3,0)}+G_{(4,2,1)}+G_{(3,3,1)}+G_{(3,2,2)}+2 \beta G_{(4,3,1)}+\beta G_{(4,2,2)}+\beta G_{(3,3,2)}+\beta^{2} G_{(4,3,2)}$. For example, the coefficient of $G_{(4,3,1)}$ counts the following $\lambda$-good tableaux of weight $(2,2,1)$ of shape $\mu$ :

$$
\begin{array}{|l|l|l|l|}
\hline 1 & 12 \\
\hline 2 & 3 \\
\hline
\end{array}, \quad \begin{array}{|l|l|}
\hline 1 & 1 \\
\hline 2 & 23 \\
\hline
\end{array}
$$

\section{Proof}

For $\alpha \in \mathbb{N}^{n}$, define $A_{\alpha}:=\operatorname{det}\left(x_{i}^{\alpha_{j}}\left(1+\beta x_{i}\right)^{j-1}\right)_{n \times n}$, and $\rho=(n-1, \ldots, 1,0)$.

Lemma 1. We have $A_{\rho}=\prod_{1 \leq i<j \leq n}\left(x_{i}-x_{j}\right)$.

Proof. In the determinantal expression for $A_{\rho}$, we can rewrite the entries of $j$ th columns of the corresponding matrix to $x_{i}^{n-j}$ inductively on $j$ (simultaneously for all $i$ ). The case $j=1$ is obvious. Let $j>1$. We assume that the $(i, k)$ entries are rewritten as $x_{i}^{n-k}$ for $1 \leq k<j$. When we expand $x_{i}^{n-j}\left(1+\beta x_{i}\right)^{j-1}$, the terms of degrees greater than $n-j$ in $x_{i}$ are eliminated by subtracting some multiples of the columns to the left of $j$ th one. Then only the term $x_{i}^{n-j}$ remains in the entry.

In order to prove Theorem 1, it suffices to prove the following.

Proposition 1. For all $\lambda \in \mathcal{P}_{n}$ and skew diagram $\theta$, we have

$$
A_{\lambda+\rho} \cdot G_{\theta}=\sum_{T \in \mathcal{T}(\theta)^{\lambda-\text { good }}} \beta^{|T|-|\theta|} A_{\lambda+\omega(T)+\rho},
$$

where $\mathcal{T}(\theta)^{\lambda \text {-good }}$ denote the set of all $\lambda$-good tableaux of shape $\theta$. 
Corollary 1 (Bi-alternant formula). For $\mu \in \mathcal{P}_{n}$, we have $G_{\mu}=A_{\mu+\rho} / A_{\rho}$.

Proof of Corollary 1. The set $\mathcal{T}(\mu)^{\emptyset \text {-good }}$ consists of only one element $T_{0}$ whose entries in row $i$ are all $i$, so that $\omega\left(T_{0}\right)=\mu$. Thus if $\lambda=\emptyset$ and $\theta=\mu$ in (3.2) then the righthand side is $A_{\mu+\rho}$, and hence we have $A_{\rho} G_{\mu}=A_{\mu+\rho}$. Then by Lemma 1 , we have the formula.

Proof of Theorem 1. Note that $\lambda+\omega(T)$ is a partition for $T \in \mathcal{T}(\theta)^{\lambda \text {-good }}$. Dividing both hand sides of (3.2) by $A_{\rho}$ and use Corollary 1, we have

$$
G_{\lambda} \cdot G_{\theta}=\sum_{T \in \mathcal{T}(\theta)^{\lambda-\text { good }}} \beta^{|T|-|\theta|} G_{\lambda+\omega(T)} .
$$

Gathering the terms of the same weight, we have the theorem.

The rest of this paper is devoted to the proof of Proposition 1.

Lemma 2. $A_{\lambda+\rho} \cdot G_{\theta}=\sum_{T \in \mathcal{T}(\theta)} \beta^{|T|-|\theta|} A_{\lambda+\omega(T)+\rho}$.

Proof. Define $\phi_{n}=\prod_{i=1}^{n}\left(1+\beta x_{i}\right)^{i-1}$. Then we have $A_{\alpha}=\pi_{n}\left(x^{\alpha} \phi_{n}\right)$ with $\pi_{n}=$ $\sum_{\sigma \in S_{n}} \operatorname{sgn}(\sigma) \sigma$, where $\sigma \in S_{n}$ permutes the variables $x_{1}, \ldots, x_{n}$. Since $G_{\theta}$ is symmetric, we have $A_{\lambda+\rho} G_{\theta}=\pi_{n}\left(G_{\theta} x^{\lambda+\rho} \phi_{n}\right)$. By using (1.1) and linearity of $\pi_{n}$ we have the result.

The proof of Proposition 1 completes if we can show the following:

$$
\sum_{T \in \mathcal{T}(\theta)^{\lambda-\mathrm{bad}}} \beta^{|T|-|\theta|} A_{\lambda+\omega(T)+\rho}=0,
$$

where we set $\mathcal{T}(\theta)^{\lambda \text {-bad }}:=\mathcal{T}(\theta)-\mathcal{T}(\theta)^{\lambda \text {-good }}$.

If $T$ is $\lambda$-bad, i.e., $T \in \mathcal{T}(\theta)^{\lambda \text {-bad }}$. Let $j_{0} \geq 1$ be the smallest integer such that $\lambda+\omega\left(c w(T)_{j_{0}}\right) \notin \mathcal{P}_{n}$. Then if $k$ is the $j_{0}$ th element of $c w(T)$, we have

$$
\lambda_{k}+\omega_{k}\left(c w(T)_{j_{0}}\right)>\lambda_{k-1}+\omega_{k-1}\left(c w(T)_{j_{0}}\right) .
$$

Let $b$ be the box in the skew Young diagram $\theta$ where the corresponding entry $k$, namely $j_{0}$ th letter of $c w(T)$, is contained. We denote $b=(r, c)$ if $b$ is in row $r$ and column $c$. Consider the leftmost box $b^{\prime}=\left(r, c^{\prime}\right)\left(c^{\prime} \leq c\right)$ in row $r$ which contains $k$. Let $T^{\prime}$ denote the labeling obtained from $T$ as follows: if $k-1$ is contained in $b^{\prime}$, we remove the entry $k-1$ from $b^{\prime}$, otherwise we add $k-1$ into $b^{\prime}$, leaving the remainder of $T$ unchanged.

Example. Let $\lambda=(3,2,2)$. Then the tableau $T$ in the Introduction is $\lambda$-bad. In fact, $\lambda+\omega\left(c w(T)_{j}\right)$ for $j=1, \ldots, 5$ can be depicted as follows:

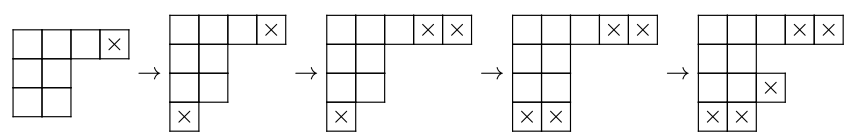


Each of the diagram but the last one is a partition. Thus $j_{0}=5, k=3, b=(2,5)$, and $b^{\prime}=(2,3)$. Let $T^{\prime}$ be the resulting labeling (tableau). We have

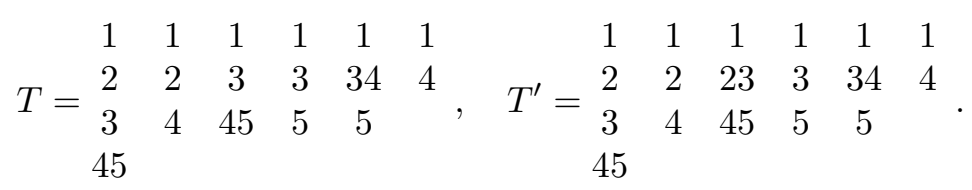

Let $T_{<c}$ denote the subtableau of $T$ formed by the entries in columns strictly left of column $c$. We use similar notations such as $T_{>c}$ and $T_{\geq c}$ in the obvious way. Note that the choice of $j_{0}$ implies that

$$
\begin{gathered}
\lambda_{k}+\omega_{k}\left(T_{\geq c}\right)=\lambda_{k-1}+\omega_{k-1}\left(T_{\geq c}\right)+1, \\
\lambda_{k}+\omega_{k}\left(T_{>c}\right)=\lambda_{k-1}+\omega_{k-1}\left(T_{>c}\right) .
\end{gathered}
$$

These equalities are true because the number of each letter occurring in a given column of $T$ is at most one, and column $c$ is the first one that violates the condition that $T_{\geq c}$ is $\lambda$-good.

Lemma 3. Let $T \in \mathcal{T}(\theta)^{\lambda \text {-bad }}$. Then the labeling $T^{\prime}$ defined above is a semistandard $\lambda$-bad tableau. Moreover, $\tau: T \mapsto T^{\prime}$ is an involution on $\mathcal{T}(\theta)^{\lambda \text {-bad }}$.

Proof. We will first check semistandardness of $T^{\prime}$. If $b^{\prime}$ contains $k-1$, we can safely remove $k-1$ from $b^{\prime}$ because there is an entry $k$ so the box in $T^{\prime}$ has a non-empty label. Semistandardness of $T^{\prime}$ is obvious. Suppose $b^{\prime}$ contains no $k-1$. For the boxes to the left, right of $b^{\prime}$ and below $b^{\prime}$, there is no problem in semistandardness when we add $k-1$ into $b^{\prime}$. In fact, each entry of the box left, right, below is smaller than $k$, larger than or equal to $k$, and larger than $k$. We claim that there is no $k-1$ in the box above $b$. If otherwise there is $k-1$ above the box $b$, then equality (3.5) cannot happen. The claim implies that there is no $k-1$ above the box $b^{\prime}$ either. Thus we can add $k-1$ into the box $b^{\prime}$ without violating semistandardness.

The resulting $T^{\prime}$ is $\lambda$-bad, because we have $c w(T)_{j_{0}}=c w\left(T^{\prime}\right)_{j_{0}}$ from the construction. We see that the map $T \mapsto T^{\prime}$ is an involution. In fact, when we make $\left(T^{\prime}\right)^{\prime}$, we pick out the same boxes $b, b^{\prime}$ in $T^{\prime}$ and remove or add $k-1$ at the same box $b^{\prime}$.

Using the involution $\tau$ we decompose $\mathcal{T}(\theta)^{\lambda \text {-bad }}$ into two disjoint subsets $\mathcal{T}(\theta)_{+}^{\lambda \text {-bad }}$ and $\mathcal{T}(\theta)_{-}^{\lambda \text {-bad }}$. If $T, T^{\prime} \in \mathcal{T}(\theta)^{\lambda \text {-bad }}, \tau(T)=T^{\prime}$, and $\left|T^{\prime}\right|=|T|+1$, then we let

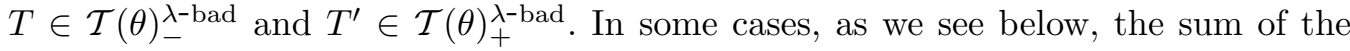
two terms of (3.3) corresponding to $T$ and $T^{\prime}$ vanishes, but in general does not. To treat general cases we use Bender-Knuth-type involutions.

Let $T \in \mathcal{T}(\theta)_{-}^{\lambda \text {-bad }}$. Chose $k$ as above so that (3.4) holds. We denote by $\sigma(T)$ the tableau obtained from $T$ by applying the Bender-Knuth involution $\sigma_{k-1}$ to $T_{<c}$, and leaving the remainder of $T$ unchanged.

Lemma 4. Let $T \in \mathcal{T}(\theta)_{-}^{\lambda \text {-bad }}$. Then we have $\sigma(T) \in \mathcal{T}(\theta)_{-}^{\lambda \text {-bad }}$. Moreover, the map $\sigma$ gives an involution on $\mathcal{T}(\theta)_{-}^{\lambda-b a d}$.

Proof. Since column $c$ has a $k$ but no $k-1$, it is easy to see that the labeling $S$ is semistandard. By the same reason as the preceding case (Lemma 3 ), $S$ is $\lambda$-bad. We will prove $S \in \mathcal{T}(\theta)_{-}^{\lambda \text {-bad }}$. Since $T \in \mathcal{T}(\theta)_{-}^{\lambda \text {-bad }}$, the box $b^{\prime}$ does not contain $k-1$. It follows that there is no box in row $r$ which contains both $k-1$ and $k$. Then by the 
definition of Bender-Knuth involution, there is no box of $S$ in row $r$ which contains both $k-1$ and $k$ either. This implies that $S \in \mathcal{T}(\theta)_{-}^{\lambda \text {-bad }}$. By a similar reason to the case of $\tau$, the map $\sigma: T \mapsto S$ is an involution.

Now the proof of (3.3) completes if we show the following:

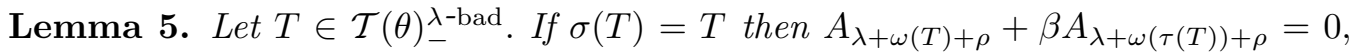
and if $\sigma(T) \neq T$ then

$$
A_{\lambda+\omega(T)+\rho}+\beta A_{\lambda+\omega(\tau(T))+\rho}+A_{\lambda+\omega(\sigma(T))+\rho}+\beta A_{\lambda+\omega(\tau \sigma(T))+\rho}=0 .
$$

Proof. Since $\omega_{k-1}(\tau(T))=\omega_{k-1}(T)+1$, we have

$$
x_{i}^{\lambda_{k-1}+\omega_{k-1}(T)+\rho_{k-1}}+\beta x_{i}^{\lambda_{k-1}+\omega_{k-1}(\tau(T))+\rho_{k-1}}=x_{i}^{\lambda_{k-1}+\omega_{k-1}(T)+\rho_{k-1}}\left(1+\beta x_{i}\right) .
$$

Therefore the sum $A_{\lambda+\omega(T)+\rho}+\beta A_{\lambda+\omega(\tau(T))+\rho}$ is a determinant of the matrix $M_{T}$ whose $(i, j)$ entry is $x_{i}^{\lambda_{j}+\omega_{j}(T)+\rho_{j}}\left(1+\beta x_{i}\right)^{j-1}$ for $j \neq k-1$, and $x_{i}^{\lambda_{k-1}+\omega_{k-1}(T)+\rho_{k-1}}(1+$ $\left.\beta x_{i}\right)^{k-1}$ for $j=k-1$ (note that the exponent of $1+\beta x_{i}$ is not $k-2$ but $k-1$ ).

Let $\alpha=\lambda+\omega\left(T_{\geq c}\right)+\rho$. Then (3.5) implies that $\alpha_{k-1}=\alpha_{k}$, i.e., $s_{k-1} \alpha=\alpha$. Since $\lambda+\omega(T)+\rho=\alpha+\omega\left(T_{<c}\right)$ and $s_{k-1} \omega\left(T_{<c}\right)=\omega\left(\sigma(T)_{<c}\right)$, we have $s_{k-1}(\lambda+\omega(T)+\rho)=$ $\lambda+\omega(\sigma(T))+\rho$. Therefore $M_{\sigma(T)}$ is obtained from $M_{T}$ by exchanging the $(k-1)$ th and the $k$ th columns. Hence the result is a consequence of the alternating property of determinants.

\section{Acknowledgments}

We thank Anders Buch, Soojin Cho, Leonardo Mihalcea, Masaki Nakagawa, Hiroshi Naruse, and Luis Serrano for helpful discussions. We also thank Kanehisa Takasaki and Tomoo Matsumura for careful reading of the manuscript. We are grateful to the referee for suggestions to improve the presentation. T.I. was partially supported by Grant-in-Aid for Scientific Research (C) 24540032.

\section{References}

[1] E.A. Bender and D.E. Knuth, Enumeration of plane partitions, J. Comb. Theory Ser. A 13 (1972), 40-54.

[2] A.S. Buch, A Littlewood-Richardson rule for the K-theory of Grassmannians, Acta. Math. 189 (2002), no. 1, 37-78.

[3] W. Fulton, "Young tableaux", Cambridge University Press, New York, 1997.

[4] A. Lascoux, Anneau de Grothendieck de la variété de drapeaux, The Grothendieck Festschrift, Vol. III, Progr. Math., Birkhäuser, Boston, 1990, 1-34.

[5] A. Lascoux and M.P. Schützenberger, Structure de Hopf de l'anneau de cohomologie et de l'anneau de Grothendieck d'une variété de drapeaux, C. R. Acad. Sci. Parix Sér. I Math., 295 (1982), 629-633.

[6] D.E. Littlewood and A.R. Richardson, Group characters and algebra, Phil. Trans. A 233 (1934), 99-141.

[7] P.J. McNamara, Factorial Grothendieck polynomials, Electron. J. Comb. 13 (2006), \#R71, 1-40.

[8] J.R. Stembridge, A concise proof of the Littlewood-Richardson rule, Electron. J. Comb. 9 (2002), \#N5, 1-4.

Department of applied mathematics, Okayama University of Science, Ridai-Cho 1-1, OKAYAMA 700-0005, JAPAN

E-mail address: ike@xmath.ous.ac.jp

E-mail address: tatsushi1023@gmail.com 
